\title{
Effects of dual-task training on balance and executive functions in Parkinson's disease: A pilot study
}

\author{
Ângela Fernandes ${ }^{a b}$, Nuno Rocha ${ }^{a}$, Rubim $\operatorname{Santos}^{c}$ \& João Manuel R. S. Tavares
}

\begin{abstract}
The aim of this study was to analyze the efficacy of cognitive-motor dual-task training compared with single-task training on balance and executive functions in individuals with Parkinson's disease. Fifteen subjects, aged between 39 and 75 years old, were randomly assigned to the dual-task training group $(n=8)$ and single-task training group $(n=7)$. The training was run twice a week for 6 weeks. The single-task group received balance training and the dual-task group performed cognitive tasks simultaneously with the balance training. There were no significant differences between the two groups at baseline. After the intervention, the results for mediolateral sway with eyes closed were significantly better for the dual-task group and anteroposterior sway with eyes closed was significantly better for the single-task group. The results suggest superior outcomes for the dual-task training compared to the single-task training for static postural control, except in anteroposterior sway with eyes closed.
\end{abstract}

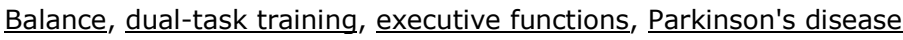

\section{Introduction}

Parkinson's disease (PD) is considered to be the second most common neurodegenerative disorder affecting currently about $1 \%$ of the world population (Andlin-Sobocki et al. 2005; Campenhausen et al. 2005; Rodrigues de Paula et al. 2006). Some projections point to a large increase in this prevalence over the next decades (Campenhausen et al. 2005).

PD is clinically defined by motor symptoms such as tremor at rest, rigidity, bradykinesia, as well as postural and gait modifications (Wielinski et al. 2005; Giroux 2007); and also by non-motor symptoms such as sleep disorders, cognitive impairment, depression, and fatigue, some of which are adverse effects of the dopaminergic medication (Hubert and Fernandez 2012). Another characteristic feature of PD is the difficulty to perform two tasks simultaneously. This difficulty is because the individuals have to focus on achieving normal movement patterns by activating the premotor cortex region without using the deficient basal ganglia circuit which is deficient in dopamine. Therefore, in dual-task situations that use the cortical resources to perform motor tasks, the performance of both the motor and cognitive components can be compromised (Wu and Hallett 2009; Brauer and Morris 2010). From this point of view, dual-task training should be considered as part of the rehabilitation process of these patients (Wu and Hallett 2009), although until now no guidelines have been defined for this type of intervention. New paradigms have been studied concerning cognitive training, such as interventions of cognitive-motor dual task. This type of intervention should be able to improve dual-task performance and/or improve motor and cognitive components individually (Silsupadol et al. 2006; K. Baker et al. 2007; Montero-Odasso et al. 2012; Yogev-Seligmann et al. 2012).

Regarding specific dual-task training, recent studies have demonstrated its efficacy in various populations such as the elderly and individuals with neurological diseases, with the most notable 
improvements in gait and balance (Silsupadol et al. 2009a, 2009b; Brauer and Morris 2010; Sethi and Raja 2012). This type of intervention for PD individuals has been focused mainly on gait (Brauer and Morris 2010; Yogev-Seligmann et al. 2011), and shows improvements in gait speed and gait variability during dual-task training. However, there is no evidence in the literature of the effects of this training on balance and executive functions evaluated independently for PD individuals. On the other hand, such separate evaluation of cognitive-motor dual-task training could be positive and enhance the meaningfulness of this type of training. Thus, considering the positive results of specific cognitive-motor dual-task training obtained in other populations and in other situations that could possibly be reproduced here, we conducted a randomized trial to study the efficacy of a cognitive-motor dual-task training program compared to a single-task program, and evaluated the cognitive and motor components independently, on PD individuals. Accordingly, we hypothesized that cognitive-motor dual-task training is more effective at improving balance and executive functions than single-task training in PD individuals.

\section{Materials and methods}

\section{Participants}

Subjects with PD were recruited from the Portuguese Association of Parkinson's Patients. The inclusion criteria used were: capacity to walk $10 \mathrm{~m}$ without gait assistance, diagnosis of PD up to Stage 3 according to the modified Hoehn and Yahr scale. The exclusion criteria used were: cognitive deficit confirmed by the Mini Mental State Examination (Folstein et al. 1975) using the following cut-off values according to the education level ( $\leq 22$ for $0-2$ years of literacy; $\leq 24$ for $3-$ 6 years; and $\leq 27$ for $\geq 7$ years (Morgado et al. 2009)), subthalamic neurosurgery, other neuromusculoskeletal and psychiatric disorders and illiteracy.

The subjects that voluntary accepted to participate were randomized to either the dual-task or single-task training group. The random assignment procedure was performed with numbers generated by a computer program (Microsoft Office Excel 2010), operated by an independent investigator. From a total of 23 eligible subjects, 20 were included in the two groups. Before the intervention program started, there were 3 dropouts in the single-task training group ( 1 for surgery, 1 due to illness, and 1 who had various absences) and 2 dropouts in the dual-task training group ( 1 for personal reasons and 1 due to illness). Hence, 7 subjects were analyzed in the single-task training group and 8 subjects in the dual-task training group. These 15 subjects made up the intervention program as shown in Figure 1. 
Figure 1. CONSORT (Schulz et al. 2010) diagram of the recruitment process adopted.

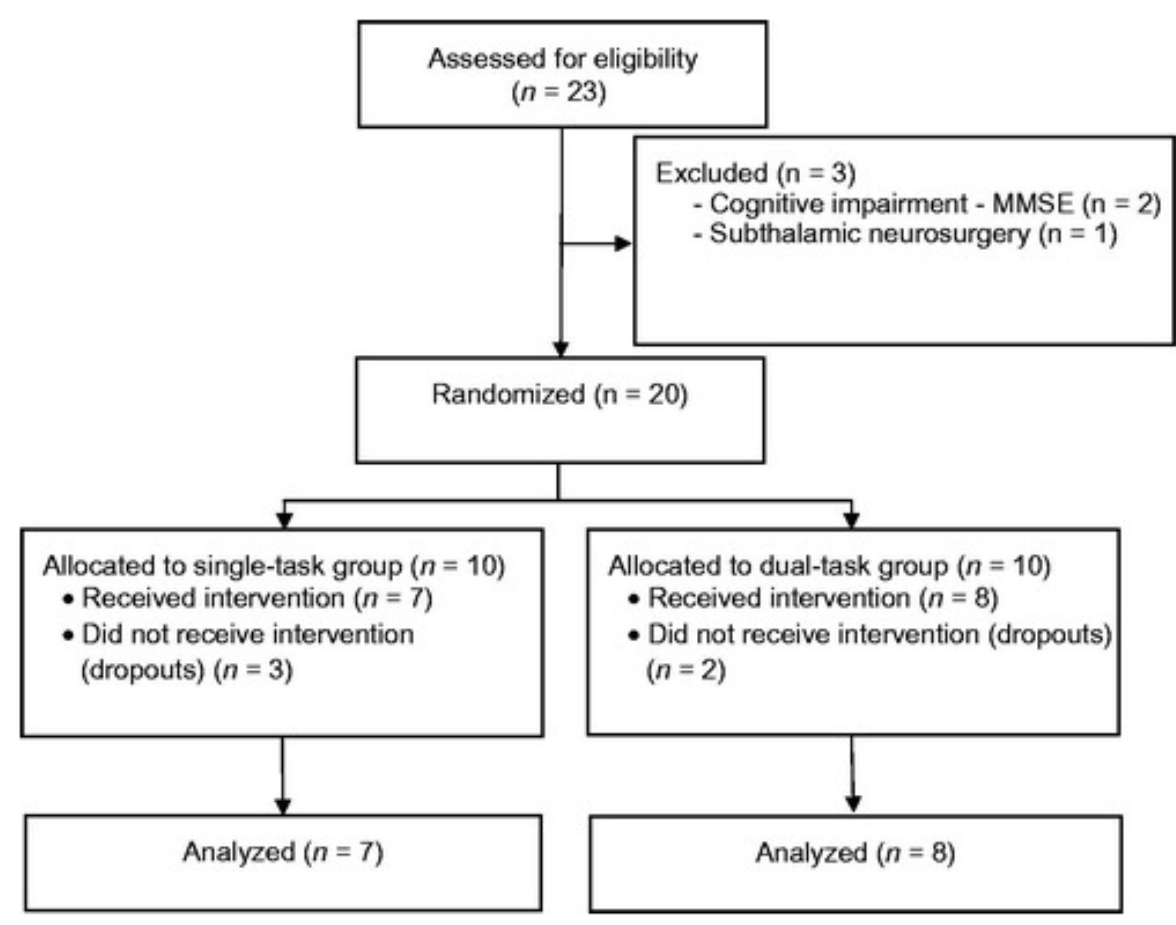

The researcher that evaluated the results was not involved in the training program and had no knowledge to which group the subjects had been assigned, in order to prevent any possible critical judgment and manipulation of the results during the evaluations. In addition, the participants were unaware of the two groups, making this a double-blind study.

The study was explained to each participant according to the intervention group in which they were randomly included. All participants gave their written informed consent in accordance to the Declaration of Helsinki, ensuring data confidentiality and freedom to withdraw from the program at any time. The study was approved by the ethics committee of "Instituto Politécnico do PortoEscola Superior de Tecnologia da Saúde" and by the directive board of "Associação Portuguesa de Doentes de Parkinson", in Portugal.

\section{Intervention}

All participants received balance training that was administered individually twice a week (60 min/session) for 6 weeks. All participants performed the same motor tasks; however, the participants of the dual-task group underwent the cognitive-motor dual-task training program and performed the cognitive tasks simultaneously with the motor tasks, while the participants of the dual-task group only underwent the single-task motor training program, and thus only performed the motor tasks. The intervention program was based on an existing training program (Silsupadol et al. 2006). The individual training sessions took place at the "Associação Portuguesa de Doentes de Parkinson" or at the "Instituto Politécnico do Porto-Escola Superior de Tecnologia da Saúde" according to each participant's preference. Each session was organized into four stations of intervention, according to Gentile's taxonomy (Gentile 2000): stability without manipulation activities (e.g., to stand on top of a foam mattress with eyes closed); gait without manipulation (e.g., walk on a narrow path); stability with handling activities (e.g., rotate the waist holding a ball); and gait manipulation activities (e.g., walking backwards around objects while holding a basket). The duration of the training sessions was the same for both groups. In the dual- 
task training, the cognitive activities included digit span (memorize a set of letters or numbers and repeat them in forward or reverse order), N-back (naming a preceding word, letter, or number to the one given by the researcher), spelling words (researcher says words to be spelled in the correct order), Stroop test (consists of two tasks, reading and naming colors; in both, the stimuli are color names printed in an incongruent color), image description (a picture is placed in front of the participant who should describe it with maximum detail), nomination (the participant must say names in a given category: flowers, animals, countries, or beginning with a letter of the alphabet), counting (counting in forward and reverse order), description of daily activities and routines (describe the activities that they normally do during a weekday or weekend and describe how to do these activities, e.g., what are the stages of taking a shower).

All participants in the dual-task group performed the same cognitive activities, but not necessarily in the same order. The complexity of the exercises was increased as the sessions progressed. This increase was based on the addition of obstacles, reduction of the pause time, increasing the complexity of the cognitive task. Each participant received individual training by a professional for 12-15 min at each station, which led to a total of $60 \mathrm{~min}$ per session. Between stations, the participants performed a transition exercise, which was getting up from and sitting down on a chair 15 times. Before beginning the exercises, all procedures were explained to the participant. No reference was made to the tasks the participant should give more importance to.

\section{Outcome measurements}

All outcome measurements were evaluated at baseline and after the intervention for all participants by a clinician who was blinded to the participant's group.

The outcome measurements of motor performance were obtained by Time Up and Go (TUG) test, Unified Parkinson's Disease Rating Scale-part III (UPDRS-III), and pressure platform.

The TUG test was used to assess the time the participant took to get up from a chair, walk $3 \mathrm{~m}$, and return to the same chair (the total distance walked was $6 \mathrm{~m}$ ) and sit down again. The time value chosen for each participant was the best, that is, the lowest value, of three trials performed (Podsiadlo and Richardson 1991). The test-retest reliability and inter-rater reliability were ICC $=0.80$ and $r=0.99$, respectively (Lim et al. 2005). UPDRS (Goetz et al. 2003) assesses the signs, symptoms, and perception of individuals concerning their performance of activities of daily living (ADLs), based on a self-report and clinical observations; it should be noted that only the motor exploration (UPDRS-III) was applied. This assessment had a high internal consistency (Cronbach's alpha $=0.96$ ) and a satisfactory inter-reliability (all items had $k>0.40$ ) (MartínezMartín et al. 1994). The pressure platform used was an Emed, from Novel (Munich, Germany), model AT 25A, with a sensorial area of $380 \times 240 \mathrm{~mm}^{2}$ and sensor resolution equal to 2 sensors $/ \mathrm{cm}^{2}$. As a stabilometric measurement, the center of pressure (COP) was evaluated in terms of the mediolateral direction (COPx), the anteroposterior direction (COPy), and the total velocity (Vt) (Ganesan et al. 2010; Holmes et al. 2010; Błaszczyk and Orawiec 2011). The participants were instructed to stand on the platform and remain in a self-selected comfortable upright position. The pressure data was taken twice: first, the subjects were instructed to remain standing on the platform and look towards a fixed point at a distance of $2 \mathrm{~m}$ for $60 \mathrm{~s}$ with their eyes open (EO); second, the subjects were instructed to remain on the same platform for the same time but now with their eyes closed (EC) (Ebersbach and Gunkel 2011). The EO/EC order 
was randomized in order to avoid any possible learning effect. The acquisition frequency of $25 \mathrm{~Hz}$ was normalized relative to each subject's body base of support.

The outcome measurements of cognitive performance were obtained by Rule Shift Cards Test (RSCardsT) and Trail Making Test (TMT) A and B. The RSCardsT is used to evaluate perseverance trends and the ability to switch from one pattern to another, by taking into account the errors and the time taken to complete the task (Golden et al. 2000). The TMT (Reitan1992) is a test divided into two parts: part $A$ evaluates attention and processing speed; and part $B$ that assesses the cognitive flexibility and sequential alternation. In each part, the final score is the total time needed to complete the task (Reitan 1992).

As in other similar studies with this type of population, all tests were carried out when the participants were taking the prescribed medication, denoted as "ON" medication (Conradsson et al. 2012; Kelly et al. 2012).

\section{Statistical analysis}

According to the nature of the variables under study, descriptive statistical analysis was performed using proportions for the variable gender, and measures of central tendency and dispersion for the variables age, education, hour of physical activity, height, weight, years of disease, and intervention outcomes.

For the inferential analysis, the Kolmogorov-Smirnov test was used to assess data normality. Since the normality of the data distribution could not be assumed, we chose to use non-parametric tests. The Mann-Whitney test for independent samples was used to verify the differences between the two groups at baseline and after intervention. In order to analyze which of the interventions was more effective, the changed scores (after the interventions relative to baseline) were used. Two-tailed tests were used in all analyses and were considered statistically significant when $p<0.05$. The training effect was calculated using Cohen's $d$ rule of thumb (Cohen 1988): low, $0.20 \leq d<0.50$; medium, $0.50 \leq d<0.80$; and high, $d \geq 0.80$. The data collected was conducted using IBM SPSS Statistics 22.0 (SPSS, Chicago, IL, USA).

\section{Results}

The values in Table I reveal that there were no significant differences between the two groups in terms of age, gender, education level, weight, height, years of illness, and number of falls. Concerning the cognitive performance, there were no significant differences between groups at baseline on the RSCardsT and TMT A and B. As to the motor performance, there were no differences between groups on UPDRS-part III, TUG, COPx, COPy, and Vt with eyes open and with eyes closed. 
Table I. Comparison at baseline between the single- and dual-task groups.

\begin{tabular}{lccc}
\hline & $\begin{array}{c}\text { Single-task } \\
\text { group }(n=8)\end{array}$ & $\begin{array}{c}\text { Dual-task } \\
\text { group }(n=7)\end{array}$ & $p$ value \\
\hline Age (years) & $62.3(12.9)$ & $63.4(9.5)$ & 0.862 \\
Gender, male (\%) & $6(85.7 \%)$ & $5(62.5 \%)$ & $0.310^{\mathrm{a}}$ \\
Education (years) & $10.4(5.1)$ & $8.6(6.4)$ & 0.288 \\
Physical activity (hours per week) & $1.9(1.3)$ & $1.3(0.3)$ & 0.208 \\
Body weight (kg) & $67.3(13.5)$ & $66.8(13.2)$ & 0.817 \\
Height (cm) & $168.3(8.0)$ & $163.9(7.4)$ & 0.121 \\
Years of disease & $7.7(7.5)$ & $8.8(4.3)$ & 0.115 \\
Time Up and Go & $11.8(4.4)$ & $11.3(3.8)$ & 0.798 \\
UPDRS-part III & $14.8(3.9)$ & $14.3(4.2)$ & 0.795 \\
Eyes opened & & & \\
Mediolateral sway (COPx-cm) & $0.938(0.457)$ & $0.813(0.249)$ & 0.848 \\
Anteroposterior sway (COPy-cm) & $1.084(0.351)$ & $1.120(0.527)$ & 0.655 \\
Total velocity (Vt-cm/s) & $0.513(0.426)$ & $0.337(0.082)$ & 0.898 \\
Eyes closed & & & \\
Mediolateral sway (COPx-cm) & $0.671(0.248)$ & $0.813(0.171)$ & 0.949 \\
Anteroposterior sway (COPy-cm) & $1.187(0.473)$ & $1.133(0.434)$ & 0.137 \\
Total velocity (Vt-cm/s) & $0.578(0.315)$ & $0.538(0.447)$ & 0.491 \\
RSCardsT & $1.71(1.38)$ & $2.25(1.49)$ & 0.475 \\
TMT A & $86.33(69.92)$ & $68.75(28.40)$ & 0.948 \\
TMT B & $186.50(98.78)$ & $168.75(55.81)$ & 0.439 \\
\hline
\end{tabular}

Results are mean and (standard deviation) or (\%).

${ }^{2}$ Chi-square test.

In order to analyze which of the interventions was more effective, the differences between the two groups were statistically analyzed after the interventions relative to baseline (Table II). In terms of the motor performance, the only differences were found in COPx and COPy with eyes closed. As to the COPx, the difference between baseline and after intervention was significantly higher for the dual-task group than for the single-task group, $U=7.5, p=0.026$, with high effect size, $d=1.094$. The difference between baseline and after intervention in terms of the COPy was significantly lower for the dual-task group than for the single-task group, $U=7.5, p=0.029$, with high effect size, $d=1.43$. Nevertheless, the Vt with eyes open and with eyes closed revealed a high effect size ( $d=0.922$ and 0.902 , respectively), and the remaining variables had a medium effect size.

Table II. Comparison between the single- and dual-task groups after the intervention relative to baseline.

\begin{tabular}{lcccc}
\hline & $\begin{array}{c}\text { Single-task } \\
\text { group }(n=8)\end{array}$ & $\begin{array}{c}\text { Dual-task } \\
\text { group }(n=7)\end{array}$ & $p$ value & Size effect \\
\hline Time Up and Go & $-1.800(1.127)$ & $-2.900(3.318)$ & 0.620 & 0.480 \\
UPDRS-part III & $-4.833(3.764)$ & $-7.000(2.204)$ & 0.345 & 0.792 \\
Eyes opened & & & & \\
Mediolateral sway (COPx-cm) & $-0.273(0.325)$ & $-0.145(0.093)$ & 0.535 & 0.581 \\
Anteroposterior sway (COPy-cm) & $-0.096(0.366)$ & $-0.273(0.257)$ & 0.848 & 0.605 \\
Total velocity (Vt-cm/s) & $-0.148(0.208)$ & $-0.012(0.091)$ & 0.128 & 0.922 \\
Eyes closed & $0.112(0.370)$ & $-0.165(0.114)$ & $0.026^{*}$ & 1.094 \\
Mediolateral sway (COPx—cm) & $-0.341(0.465)$ & $0.286(0.479)$ & $0.029^{*}$ & 1.430 \\
Anteroposterior sway (COPy-cm) & $-0.130(0.365)$ & $0.096(0.176)$ & 0.181 & 0.902 \\
Total velocity (Vt-cm/s) & $0.286(0.489)$ & $1.125(2.031)$ & 0.336 & 0.590 \\
RSCardsT & $-11.833(43.190)$ & $-2.750(15.416)$ & 0.950 & 0.324 \\
TMT A & $-31.333(48.980)$ & $-0.250(32.115)$ & 0.345 & 0.839 \\
TMT B & & & & \\
\hline
\end{tabular}

Results are mean and (standard deviation).

${ }^{*} p$-value $<0.05$.

No significant differences were found between the two groups in terms of the executive functions performed. However, the TMT B had a high effect size $(d=0.839)$, the RSCardsT presented a medium effect size $(d=0.590)$, and the TMT A had a small effect size $(d=0.324)$. 


\section{Discussion}

Studies have reported the positive influence of targeted interventions for motor training, whether for different cognitive components, including level of attention, processing speed, flexibility and alternating sequential, or for neuromotor issues, mainly in terms of muscle resistance, coordination, balance, and agility (Moher et al. 2009; Tanaka et al. 2009; L. Baker et al.2010; Mirelman et al. 2011; Davis et al. 2013; Tabak et al. 2013). Our research has demonstrated that in a cognitive-motor dual-task training program with 12 sessions, the dual-task training was only statistically more effective than the single-task training for the COPx with eyes closed. A lower oscillation, that is, smaller COP displacements, corresponds to a higher postural stability (Mochizuki et al. 2006) and thus, in agreement, our results suggested a better balance after the intervention program in the dual-task training group. As to COPy with closed eyes, significant differences were also found, but the dual-task training group presented values worse than the single-task training group. This fact can be explained by the number of years of the disease that was higher in the dual-task training group. The COP of these participants was shifted to a more posterior position in order to compensate the usual postural deformities caused by high muscular rigidity (Jankovic 2008; Matinolli 2009). This body position, together with the loss of postural reflexes, age-related sensory changes, as well as other features, leads to greater instability in the anteroposterior component (Jankovic 2008).

COPx and COPy values with eyes open did not show significant differences between the two groups, but these variables had lower values after intervention in both. Some authors, for example, Oie et al. (2002) and Tjernström et al. (2002), defend that vision provides important feedback to the subjects about the physical environment, their spatial interactions and body sway, which complements the information provided by other sensorial receivers. Thus, the eyes open provides important information about postural orientation and helps to optimize the balance control, which may explain the better results found for COP displacement under this condition.

With regard to the $\mathrm{Vt}$, it was found that the results were not statistically significant, but the effect size was high, as in previous studies with elderly individuals (Li et al. 2010; Plummer-D'Amato et al. 2012). Mochizuki et al. (2006) suggested that the lower values of velocity correspond to higher postural stability; however, in our study, the Vt with eyes closed increased in the dual-task training group, which may be a mechanism to compensate for the lower oscillation.

Based on the TUG test as well as the UPDRS-III test, the difference in terms of mobility was higher in the dual-task training group, with medium effect size, which indicates an improvement of the functional mobility of the individuals. These findings are consistent with other studies in which the average values were better in dual-task training programs, but with no significant results (Vaillant et al. 2006; Her et al. 2011; Jiejiao et al. 2012; Plummer-D'Amato et al. 2012).

Regarding the cognitive components, the TMT A, TMT B, and RSCardsT results showed a tendency for improvement in both groups after intervention, likewise in a previous study by Hiyamizu et al. (2011) with healthy elderly individuals. These findings are also in agreement with other studies where visible improvements after dual-task interventions were found, although without statistical significance (Pellecchia 2005; Silsupadol et al. 2009a; Makizako et al. 2012; Pedroso et al. 2012).

The present study, as far as the authors know, is innovative as it is the first study to assess the outcomes of a dual-task intervention on balance and executive functions in subjects with PD. 
Nonetheless, there are some limitations that should be discussed. The small size of the studied sample can limit the results, particularly regarding the significance of the statistical tests performed and the generalization of the findings. Hence, this work should be considered as a pilot study that has added knowledge concerning the effects of dual-task training on balance and executive functions in patients with PD. All participants involved were "ON" cholinergic medication, but the effect of the medication on the participants' performance was not taken into account. Therefore, although the intervention adopted was selected based on other closely related studies (Silsupadol et al. 2009a, 2009b), it is suggested that future studies should also include a cognitive training before or after the balance training in the group that undergo the single-task training.

In conclusion, as was hypothesized for this study, our findings revealed a more positive response with the dual-task intervention compared to the single-task intervention. The motor training with a cognitive task performed simultaneously improved the performance of some parameters related to balance and executive functions of individuals with PD. These observations highlight the strength of rehabilitative interventions based on dual-task training.

\section{Acknowledgement}

This research was carried out with the support and contribution of the first Author's PhD grant from "Instituto Politécnico do Porto and Escola Superior de Tecnologia da Saúde", in Portugal.

\section{References}

1. Andlin-Sobocki P, Jonsson B, Wittchen H, Olesen J. 2005. Cost of disorders of the brain in Europe. Eur J Neurol 12(1):1-27

2. Baker K, Rochester L, Nieuwboer A. 2007. The immediate effect of attentional, auditory and a combined cue strategy on gait during single and dual tasks in Parkinson's disease. Arch Phys Med Rehabil 88(12):1593-1600

3. Baker L, Frank L, Foster-Scgubert K, Green P, Wilkinson C, McTiernan A, Craft S. 2010. Effects of aerobic exercise on mild cognitive impairment: A controlled trial. Arch Neurol 67(1):71-79

4. Błaszczyk JW, Orawiec R. 2011. Assessment of postural control in patients with Parkinson's disease: Sway ratio analysis. Hum Mov Sci 30(2):396-404

5. Brauer SG, Morris ME. 2010. Can people with Parkinson's disease improve dual tasking when walking? Gait Posture 31:229-233

6. Campenhausen S, Bornschein B, Wick R, Botzel K, Sampaio C, Poewe W. 2005. Prevalence and incidence of Parkinson's disease in Europe. Eur Neuropsychopharmacol 15:473-490

7. Cohen J. 1988. Statistical power analysis for the behavioral sciences. 2nd ed. Hillsdale, NJ: Lawrence Erbaum

8. Conradsson D, Löfgren N, Ståhle A, Hagströmer M, Franzén E. 2012. A novel conceptual framework for balance training in Parkinson's disease-Study protocol for a randomised controlled trial. BMC Neurol 12:1-11

9. Davis J, Bryan S, Marra C, Sharma D, Chan A, Beattie L, Liu-Ambrose T. 2013. An economic evaluation of resistance training and aerobic training versus balance and toning exercises in older adults with mild cognitive impairment. PLOS One 8(5):1-9 
10. Ebersbach G, Gunkel M. 2011. Posturography reflects clinical imbalance in Parkinson's disease. Mov Disord 26(2):241-246

11. Folstein MF, Folstein SE, McHugh PR. 1975. "Mini-mental state". A practical method for grading the cognitive state of patients for the clinician. J Psychiatr Res 12(3):189-198

12. Ganesan M, Pal PK, Gupta A, Sathyaprabha TN. 2010. Dynamic posturography in evaluation of balance in patients of Parkinson's disease with normal pull test: Concept of a diagonal pull test. Parkinsonism Related Disord 16(9):595-599

13. Gentile AM. 2000. Skill acquisition: Action, movement and neuromotor processes. 2nd ed. Rockville: Aspen

14. Giroux ML. 2007. Parkinson disease: Managing a complex, progressive disease at all stages. Cleve Clin J Med 74(5):313-328

15. Goetz CG, Poewe W, Rascol O, Sampaio C, Stebbins GT, Fahn S, Seidl L. 2003. The Unified Parkinson's Disease Rating Scale (UPDRS): Status and recommendations. Mov Disord 18(7):738750

16. Golden C, Espe-Pfeifer P, Wachsler-Felder J. 2000. Neuropsychological interpretations of objective psychological tests. New York: Plenum

17. Her JG, Park KD, Yang Y, Ko T, Kim H, Lee J, Woo JH, Ko J. 2011. Effects of balance training with various dual-task conditions on stroke patients. J Phys Ther Sci 23(5):713-717

18. Hiyamizu M, Morioka S, Shomoto K, Shimada T. 2011. Effects of dual task balance training on dual task performance in elderly people: A randomized controlled trial. Clin Rehabil 26(1):58-67. doi: $10.1177 / 0269215510394222$

19. Holmes J, Jenkins M, Johnson A, Adams S, Spaulding S. 2010. Dual-task interference: The effects of verbal cognitive tasks on upright postural stability in Parkinson's disease. Parkinson's Dis 69(6):4952

20. Hubert H, Fernandez M. 2012. Nonmotor complications of Parkinson disease. Cleve Clin J Med 79:14-18

21. Jankovic J. 2008. Parkinson's disease: Clinical features and diagnosis. J Neurol Neurosurg Psychiatry 79:368-376

22. Jiejiao Z, Xueqiang W, Yueying X, Ying Y, Liyan S, Zhenwen L. 2012. Cognitive dual-task training improves balance function in patients with stroke. HealthMED 6(3):840-845

23. Kelly V, Eusterbrock A, Shumway-Cook A. 2012. A review of dual-task walking deficits in people with Parkinson's disease: Motor and cognitive contributions, mechanisms, and clinical implications. J Parkinson's Dis 1-14

24. Li KZ, Roudaia E, Lussier M, Bherer L, Leroux A, McKinley PA. 2010. Benefits of cognitive dualtask training on balance performance in healthy older adults. J Gerontol A Biol Sci Med Sci 65(12):1344-1352

25. Lim LI, van Wegen EE, de Goede CJ, Jones D, Rochester L, Hetherington V, Nieuwboer A, Willems AM, Kwakkel G. 2005. Measuring gait and gait-related activities in Parkinson's patients' own home environment: A reliability, responsiveness, and feasibility study. Parkinsonism Related Dis 11:19-24

26. Makizako H, Doi T, Shimada H, Yoshida D, Tsutsumimoto K, Uemura K, Suzuki T. 2012. Does a multicomponent exercise program improve dual-task performance in amnestic mild cognitive impairment? A randomized controlled trial. Aging Clin Exp Res 24(6). doi: 10.3275/8760

27. Martínez-Martín P, Gil-Nagel A, Gracia LM, Gómez JB, Martínez-Sarriés J, Bermejo F. 1994. Unified Parkinson's Disease Rating Scale characteristics and structure. Mov Disord 9(1):76-83 
28. Matinolli M. 2009. Balance, mobility and falls in Parkinson's disease. Oulu: University of Oulu

29. Mirelman A, Maiden I, Herman T, Deutsch JE, Giladi N, Hausdorff JM. 2011. Virtual reality for gait training: Can it induce motor learning to enhance complex walking and reduce fall risk in patients with Parkinson's disease? J Gerontol A Biol Sci Med Sci 66:234-240

30. Mochizuki L, Duarte M, Amadio AC, Zatsiorsky VM, Latash ML. 2006. Changes in postural sway and its fractions in conditions of postural instability. J Appl Biomech 22(1):51-60

31. Moher D, Liberati A, Tetzlaff J, Altman D. 2009. Preferred reporting items for systematic reviews and meta-analyses: The PRISMA statement. PLOS Med 6(7):1-6

32. Montero-Odasso M, Verghese J, Beauchet O, Hausdorff J. 2012. Gait and cognition: A complementary approach to understanding brain function and the risk of falling. Am Geriatrics Soc 60(11):2127-2136

33. Morgado J, Rocha CS, Maruta C, Guerreiro M, Martins IP. 2009. New normative values of MiniMental State Examination. Sinapse 9(2):19-25

34. Oie K, Kiemel T, Jeka J. 2002. Multisensory fusion: Simultaneous re-weighting of vision and touch for the control of human posture. Brain Res Cogn Brain Res 14(1):164-176

35. Pedroso RV, Coelho FGM, Santos-Galduróz RF, Costa JLR, Gobbi S, Stella F. 2012. Balance, executive functions and falls in elderly with Alzheimer's disease (AD): A longitudinal study. Arch Gerontol Geriatrics 54(2):348-351

36. Pellecchia GL. 2005. Dual-task training reduces impact of cognitive task on postural sway. J Mot Behav 37(3):239-246

37. Plummer-D'Amato P, Cohen Z, Daee NA, Lawson SE, Lizotte MR, Padilla A. 2012. Effects of once weekly dual-task training in older adults: A pilot randomized controlled trial. Geriatr Gerontol Int 12(4):622-629

38. Podsiadlo D, Richardson S. 1991. The timed "Up \& Go": A test of basic functional mobility for frail elderly persons. J Am Geriatr Soc 39(2):142-148

39. Reitan RM. 1992. Trail making test: Manual for administration and scoring. New York: Tuscan

40. Rodrigues de Paula F, Teixeira-Salmela L, Faria C, Brito P, Cardoso F. 2006. Impact of an exercise program on physical, emotional, and social aspects of quality of life of individuals with Parkinson's disease. Mov Disord 21:1073-1077

41. Schulz KF, Altman DG, Moher D. 2010. CONSORT 2010 statement: Updated guidelines for reporting parallel group randomized trials. Ann Intern Med 152:726-732

42. Sethi V, Raja R. 2012. Effects of dual task training on balance and activities of daily livings in patients with Parkinsonism. Int J Neurol Med Res 3(1):1359-1364

43. Silsupadol P, Siu KC, Shumway-Cook A, Woollacott M. 2006. Training of balance under singleand dual-task conditions in older adults with balance impairment. Phys Ther 86(2):269-281

44. Silsupadol P, Lugade V, Shumway-Cook A, van Donkelaar P, Chou LS, Mayr U, Woollacott $\mathrm{MH}$. 2009a. Training-related changes in dual-task walking performance of elderly persons with balance impairment: A double-blind, randomized controlled trial. Gait Posture 29(4):634-639

45. Silsupadol P, Shumway-Cook A, Lugade V, van Donkelaar P, Chou LS, Mayr U, Woollacott $\mathrm{MH}$. 2009b. Effects of single-task versus dual-task training on balance performance in older adults: A double-blind, randomized controlled trial. Arch Phys Med Rehabil 90(3):381-387. doi: 10.1016/j.apmr.2008.09.559

46. Tabak R, Aquije G, Fisher B. 2013. Aerobic exercise to improve executive function in Parkinson disease: A case series. J Neurol Phys Ther 37:58-64 
47. Tanaka K, Quadros A, Santos R, Stella F, Gobbi L, Gobbi S. 2009. Benefits of physical exercise on executive functions in older people with Parkinson's disease. Brain Cogn 69:435-441

48. Tjernström F, Fransson P, Hafström A, Magnusson M. 2002. Adaptation of postural control to perturbations_A process that initiates long-term motor memory. Gait Posture 15(1):75-82

49. Vaillant J, Vuillerme N, Martigné P, Caillat-Miousse JL, Parisot J, Nougier V, Juvin R. 2006. Balance, aging, and osteoporosis: Effects of cognitive exercises combined with physiotherapy. Joint Bone Spine 73(4):414-418

50. Wielinski CL, Erickson-Davis C, Wichmann R, Walde-Douglas M, Parashos SA. 2005. Falls and injuries resulting from falls among patients with Parkinson's disease and other Parkinsonian syndromes. Mov Disord 20:410-415

51. Wu T, Hallett M. 2009. Dual task interference in Parkinson's disease. Touch Briefings 34-37

52. Yogev-Seligmann G, Giladi N, Brozgol M, Hausdorff J. 2011. A training program to improve gait while dual tasking in patients with Parkinson's disease: A pilot study. Arch Phys Med Rehabil 20:176181

53. Yogev-Seligmann G, Rotem-Galili Y, Dickstein R, Giladi N, Hausdorff J. 2012. Effects of explicit prioritization on dual task walking in patients with Parkinson's disease. Gait Posture 35(4):641-646 